\title{
A semi-anaytical model for the analysis of a Permanent Magnet Tubular Linear Generator
}

\author{
Antonino Musolino, Marco Raugi, Rocco Rizzo and Luca Sani \\ Dipartimento di Ingegneria dell'Energia, dei Sistemi, del Territorio e delle Costruzioni, University of Pisa, Italy \\ \{antonino.musolino, marco.raugi, rocco.rizzo, luca.sani\}@ unipi.it
}

\begin{abstract}
In this paper the authors introduce a semi-analytical model for the analysis and the design of a Permanent Magnet (PM) tubular linear generator intended for electrical energy generation from sea waves. The translator of the analyzed machine is constituted by axially magnetized ferrite PMs with alternating polarity and soft-magnetic pole-pieces in between; a two poles, double layer three-phase winding is located in the slots of the stator. The presented model, based on use of the Carter coefficient and of the Fourier transform in the direction of the motion, is able to take into account the end effects due to the finite length of the stator. The presence of slots and teeth is subsequently considered by some post processing calculation carried on the results of the semi-analytical model. Comparison with a Finite Element analysis and with measurements taken on a prototype has been performed to validate the presented model. The model can be easily extended to other translator typologies, e.g. to air core translator with Halbach array of NdFeB PMs.
\end{abstract}

Keywords-analytical model, tubular machine, permanent megnet, hard ferrites, soft ferrites, Finite Elements Method.

\section{INTRODUCTION}

The marine energy is considered as one of the most interesting and promising sources in the worldwide renewable energies scenario [1] - [9]. It is available in several forms, such as marine streams, tidal, temperature gradient, and waves. Particularly, the energy from the waves is characterized by high power density and widespread availability.

In the recent past, tens of different devices using the wave motion were developed; some of these have already reached a full-scale demonstration stage and currently are moving toward a marketing phase, while a number of projects is under theoretical investigation and/or experimental validation.

Fig. 1 shows the general structure of the wave energy converter developed by the Swedish company Seabased [10]. When the horizontal dimension of the buoy are small with respect to the wavelength of the incident sea waves, the WEC is called point absorber. The heave motion impressed to the buoy is converted in linear motion for driving an electrical generator.

As shown in the figure the use of linear synchronous generators is the natural choice for this kind of applications [11]-[17]. These generators consist of a fixes armature and of a translator, made of PMs and iron, which serves to reduce the reluctance of the magnetic circuits. Ironless configurations are also available especially when Halbach arrays, are adopted. One of the key components of the generation system is the linear machine. A proper optimal design of the electric generator is essential for the achievement of a good efficiency.

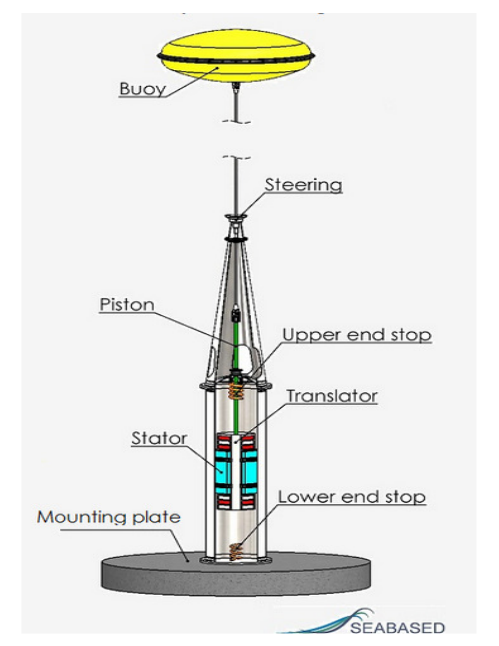

Figure 1. General structure of a wave energy converter (courtesy of Seabased Group).

Recent publications on optimization of linear generators are mostly based on equivalent network models, which are fast but may suffer of limited accuracy especially in presence of complex flux paths.

Numerical tools are able to provide detailed descriptions of the device also considering complex nonlinear phenomena such as hysteresis [18]-[20], but are usually time consuming and are not effective to insight the dependence of the performance on the design parameters.

In this work, the authors present a fast and accurate semianalytical model, capable to take into account both the entry and exit effects without the need of correction coefficients.

The paper is organized as follows: Sect. II introduces the semi analytical model of the generator [21]; while sect. III briefly discusses the post processing activities in order to take into account some effects of the presence of the slot and of the teeth. Section IV describes a prototype of the machine, and finally section $\mathrm{V}$ reports the comparison of the results obtained by the proposed model with a numerical FEM analysis and with the measurements taken on the prototype.

\section{The Semi-Analytical Model}

Fig. 2 reports a cut of the analyzed generator. The translator is on the left side of the figure and is composed of axially magnetized ferrite PMs with alternating polarity with softmagnetic pole-pieces in between. In particular, in the analyzed device, three PMs with the same polarity (yellow) are stacked with three soft ferrite rings (green) and with three PMs with 
reversed polarity w.r.t. the previous ones (red); then there are three other soft ferrite rings and so on. A three phase winding with the same number of pole pairs (two) is arranged in double layers in the 24 stator slots. Each layer has 42 turns.

The geometrical parameters of the machine are listed in table I.

TABLE I. Geometrical parameters of the machine

\begin{tabular}{|l|l|l|l|}
\hline Parameter & Symbol & value & unit \\
\hline airgap & $\delta_{a}$ & 3.0 & $\mathrm{~mm}$ \\
\hline internal radius of PMs & $r_{i, M}$ & 27.2 & $\mathrm{~mm}$ \\
\hline external radius of PMs & $r_{e, M}$ & 51.7 & $\mathrm{~mm}$ \\
\hline height of PMs & $h_{M}$ & 12.5 & $\mathrm{~mm}$ \\
\hline remanence of PMs & $B_{r}$ & 0.39 & $\mathrm{~T}$ \\
\hline coercivity & $H_{c}$ & 270.0 & $\mathrm{kA} / \mathrm{m}$ \\
\hline internal radius of stator & $R_{i, \mathrm{~S}}$ & 54.7 & $\mathrm{~mm}$ \\
\hline external radius of stator & $R_{e, \mathrm{~S}}$ & 128.0 & $\mathrm{~mm}$ \\
\hline stator slot depth & $\delta_{r, S}$ & 53.0 & $\mathrm{~mm}$ \\
\hline stator slot height & $\delta_{\mathrm{z}, S}$ & 5.5 & $\mathrm{~mm}$ \\
\hline stator length & $L$ & 324.0 & $\mathrm{~mm}$ \\
\hline pole pitch & $\tau_{p}$ & 80.0 & $\mathrm{~mm}$ \\
\hline
\end{tabular}

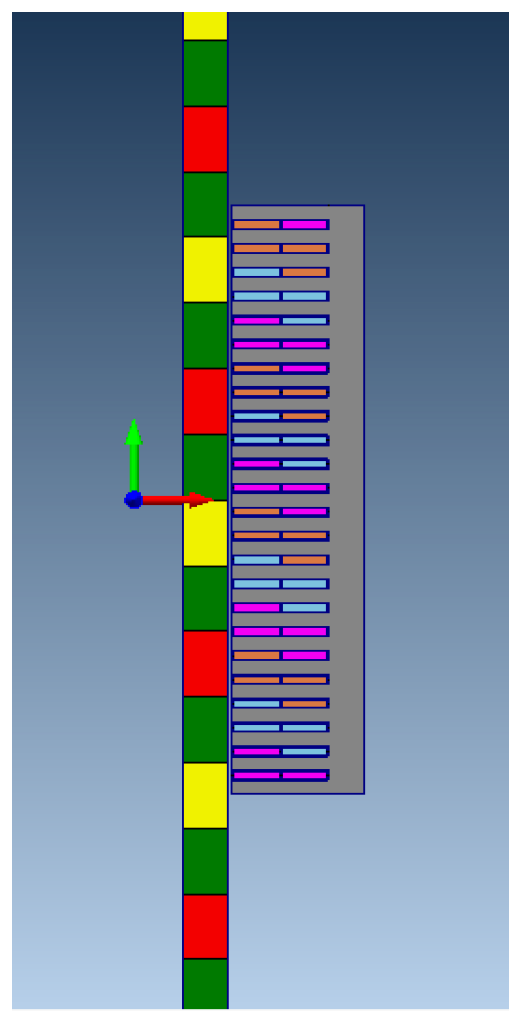

Figure 2. Cut of the PM linear generator.

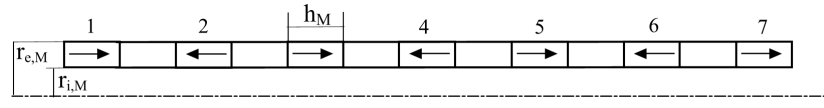

(a)

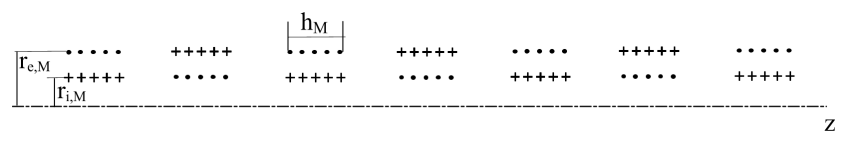

(b)

Figure 3. (a) Magnetization pattern; (b) Equivalent current distribution.

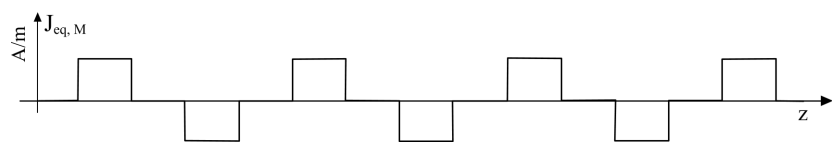

(a)

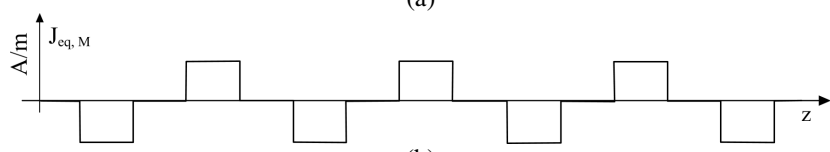

(b)

Figure 4. Equivalent current distribution: (a) $r=r_{e, M}$; (b) $r=r_{i, M}$.

An accurate analysis of the device, taking into account the movement of the translator with respect to the stator requires a numerical model with consequent long simulation times often resulting in unviable automatic design procedures [22], [23]. Faster analyses can be performed by approximated analytical models, which usually discard the end effects and the presence of the slots.

However, in the proposed semi analytical model, end effects are taken into account assuming an indefinite axial extension of both the stator and the translator, while the stator currents occupy a portion of finite length of the stators. Once the solution of the semi analytical model has been obtained, the slot leakage flux and average distribution of flux density in the teeth are evaluated by approximated formulas [24]-[25]. The effects of the presence of the slots can be taken into account by introducing the Carter coefficient [26] and equivalent current sheets.

The model of the device is built in two steps. Preliminarily a no load simulation, taking into account the iron losses in the stator is performed in order to evaluate the induced e.m.f. at the terminals of the generator. Subsequently, a balanced thee phase current system is injected in the stator windings, with the equivalent magnetization currents on the PMs set to zero, with the aim of evaluating the internal impedance of the generator.

When performing the evaluation of the no load voltage, the system of PMs on the translator is substituted by a system of equivalent magnetization currents. The axially magnetized PMs is substituted by a system of currents flowing in the inner and outer cylindrical surfaces of the rings.

Fig. 3a shows the magnetization pattern of the PMs array, while Fig. $3 b$ shows the equivalent magnetization current distribution under the hypothesis of uniform axial magnetization. The plus signs ("+") indicate entering currents, while points (“•”) indicate outgoing currents. 


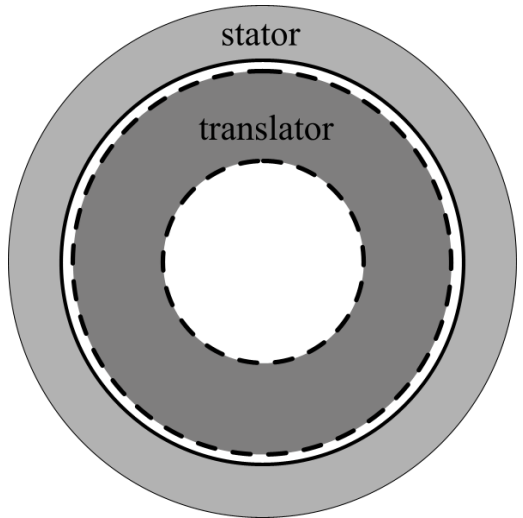

Figure 5. Cross section of the generator after the Carter procedure has been applied, and the PMs are substituted by their equivalent currents sheets.

The waveforms of the equivalent surface magnetization currents corresponding to the axially magnetized PMs are shown in Figs. $4 \mathrm{a}$ and $4 \mathrm{~b}$. The first one shows the equivalent magnetization current distribution at $r=r_{e, M}$, while the other at $r=r_{i, M}$. In these figures the entering currents (indicated with the cross) are positive.

The equivalent currents waveforms shown in Fig. 4, are periodic in the axial direction, so they can be expanded in Fourier series. For both the general expression is:

$$
J_{M, q}(r, z)=\boldsymbol{\varphi} \delta\left(r-r_{q}\right) \sum_{i=1}^{\infty} J_{i, q} \cos \left(i \frac{2 \pi}{\lambda} z+\varphi_{i, q}\right)
$$

where $\lambda$ is the wavelength of the equivalent current distributions (i.e. twice the pole pitch $\tau_{p}$ ), $\boldsymbol{\varphi}$ represents the unit vector in the azimuth direction, $r_{1}=r_{i, M} \quad r_{2}=r_{\mathrm{e}, M}$ and $\delta(r)$ is the Dirac function and $q=1,2 ; J_{i, q}$ and $\varphi_{i, q}$ are respectively the amplitude and the phase of the i-th harmonic.

We assume that these current distributions move with respect to the stator at a constant speed in the axial direction. The travelling waves of equivalent currents can be written as:

$$
\begin{gathered}
J_{M, q}(r, z, t)=\boldsymbol{\varphi} \delta\left(r-r_{q}\right) \sum_{i=1}^{\infty} J_{\mathrm{i}, q} \cos \left(k_{i}(z-v t)+\varphi_{\mathrm{i}, q}\right)= \\
\boldsymbol{\varphi} \delta\left(r-r_{q}\right) \sum_{i=1}^{\infty} J_{\mathrm{i}, q} \cos \left(2 \pi i\left(\frac{z}{\lambda}-\frac{t}{T}\right)+\varphi_{\mathrm{i}, q}\right)
\end{gathered}
$$

where $T=\lambda / v$ is the time needed to the translator to travel a distance equal to the wavelength and $k_{i}=2 \pi i / \lambda$.

Fig. 5 shows a cross section of the generator after that the Carter procedure has been used to substitute the slotted stator with a smoothed one. In this figure the dashed circular lines indicate the position of the PMs equivalent current sheets attached to the translator, whose distributions along the axial direction are shown in Figs. 4 (a) and (b).

The solid line attached to the inner surface of the stator indicates the position of the current sheet equivalent to the stator currents used to evaluate the internal impedance of the generator. The waveform of this current sheet is:

$\boldsymbol{J}_{\text {stat }}(r, z, t)=\boldsymbol{\varphi} \delta\left(r-r_{S}\right) \cos (k z-\omega t) u\left(z-\frac{L}{2}\right) u\left(\frac{L}{2}-z\right)$

where $k=2 \pi p / L, p$ is the number of pole pairs, $L$ is the length of the stator; $r_{S}$ is the radius where the stator current sheet is located considering the correction due to the Carter factor, and $u(z)$ is the unit step function.

The machine is subdivided in a number of concentric regions. The outer is vacuum, the light gray one is laminated iron with an equivalent conductivity $\sigma$ which takes into account the eddy current losses. The inner region between the equivalent magnetization current sheets takes into account of the presence of both the hard and soft ferrites. As known, once the equivalent magnetization currents are considered, the PMs are substituted by a linear material which is characterized by a magnetic permeability obtained by considering the slope of the $\mathrm{B}-\mathrm{H}$ characteristic of the PMs at the working point. We assume that this material is characterized by the same permeability as the soft ferrite and by a negligible conductivity.

The thickness of the stator in the radial direction is the same as the back iron $\left(\Delta r_{b i}=20.3 \mathrm{~mm}\right)$.

The axisymmetric semi-analytical model of the device is briefly summarized here; details are in [25].

The governing equation in terms of magnetic vector potential $\boldsymbol{A}$ constrained by the Coulomb gauge $(\nabla \cdot \boldsymbol{A}=0)$ for linear isotropic medium moving with velocity $\boldsymbol{v}$, is written as:

$$
\nabla^{2} \boldsymbol{A}=\mu \sigma\left(\frac{\partial}{\partial t} \boldsymbol{A}-\boldsymbol{v} \times(\nabla \times \boldsymbol{A})\right)
$$

where $\sigma$ and $\mu$ respectively indicate the conductivity and the permeability of the medium.

The current sheets as in (2) can be expressed as

$$
\boldsymbol{J}_{M, q}(r, z, t)=\boldsymbol{\varphi} \sum_{i=1}^{\infty} \operatorname{Re}\left\{J_{i, q} e^{j\left(i \left(z / \lambda^{\left.-\omega t)+\varphi_{1, q}\right)}\right.\right.}\right\} \delta\left(r-r_{q}\right)
$$

where $q=1,2$; spans the current sheets as shown in Fig. 5 in dashed lines. A similar expression is obtained for $\boldsymbol{J}_{\text {stat }}$ in terms of its Fourier transform.

In axisymmetric geometry and with the given currents we can write: $\boldsymbol{A}=\boldsymbol{\varphi} \tilde{A}_{\varphi}(r, z) e^{j \omega t}$ where tilde denotes phasors.

Discarding $e^{j \omega t}$, after some manipulation (4) is written as:

$$
\frac{\partial^{2} \tilde{A}_{\varphi}}{\partial r^{2}}+\frac{1}{r} \frac{\partial \tilde{A}_{\varphi}}{\partial r}+\frac{\partial^{2} \tilde{A}_{\varphi}}{\partial z^{2}}-\frac{\tilde{A}_{\varphi}}{r^{2}}=\mu \sigma\left(j \omega \tilde{A}_{\varphi}+v_{z} \frac{\partial \tilde{A}_{\varphi}}{\partial z}\right)
$$

Let $\boldsymbol{A}_{\varphi}(r, \zeta)$ be the Fourier transform of $\tilde{A}_{\varphi}(r, z)$ :

$$
\tilde{A}_{\varphi}(r, z)=\frac{1}{2 \pi} \int_{-\infty}^{\infty} \boldsymbol{A}_{\varphi}(r, \zeta) e^{-j \zeta z} d \zeta
$$

substituting in (6) and discarding $e^{-j \zeta z}$ yields: 


$$
\frac{\partial^{2} \boldsymbol{A}_{\varphi}^{(l)}}{\partial r^{2}}+\frac{1}{r} \frac{\partial \boldsymbol{A}_{\varphi}^{(l)}}{\partial r}-\left(h_{l}^{2}+\frac{1}{r^{2}}\right) \boldsymbol{A}_{\varphi}^{(l)}=0
$$

where the superscript $l$ denotes the layers as in Fig. 5, $(l=1, . ., 5 ;)$. In nonconductive material (air and translator) we have $h_{l}^{2}=\zeta^{2}$, while in conductors (stator iron) $h_{l}^{2}=j \zeta^{2}\left(s v_{s} \sigma \mu / \zeta-j\right)$, where $v_{s}=\omega / \zeta$ is the synchronous speed and $s=\left(v_{s}-v_{z}\right) / v_{s}$ is the slip.

The general solution of (8) is:

$$
\boldsymbol{A}_{\varphi}^{(l)}(r, \zeta)=C_{l} I_{1}\left(h_{l} r\right)+C_{l}^{\prime} K_{1}\left(h_{l} r\right)
$$

where $I_{1}$ and $K_{1}$ are the modified Bessel functions of first and second kind respectively.

Because of (7), the normal component (radial) of the flux density is given by:

$$
\begin{aligned}
\tilde{B}_{r}^{(l)}(r, z)= & \frac{1}{2 \pi} \int_{-\infty}^{\infty}\left[\nabla \times\left(\boldsymbol{A}_{\varphi}^{(l)}(r, \zeta) e^{-j \zeta z}\right)\right] d \zeta= \\
& \frac{1}{2 \pi} \int_{-\infty}^{\infty} \boldsymbol{B}_{r}^{(l)}(r, \zeta) e^{-j \zeta z} d \zeta
\end{aligned}
$$

with:

$$
\mathbf{B}_{r}^{(l)}(r, \zeta)=j \zeta\left(C_{l} I_{1}\left(h_{l} r\right)+C_{l}^{\prime} K_{1}\left(h_{l} r\right)\right)
$$

Similarly, the tangential (axial) component of the magnetic field strength can be written as:

$$
\boldsymbol{H}_{r}^{(l)}(r, \zeta)=\frac{h_{l}}{\mu}\left(C_{l} I_{0}\left(h_{l} r\right)-C_{l}^{\prime} K_{0}\left(h_{l} r\right)\right)
$$

Because of the asymptotic behavior of $I_{1}$ and $K_{1}$, we have $C_{\text {inner }}^{\prime}=C_{\text {outer }}=0$, while the other unknown integration coefficients are evaluated by imposing the boundary conditions at the interfaces between the layers:

$\boldsymbol{B}_{\mathrm{r}}^{(l)}\left(\mathrm{r}_{l}, \zeta\right)=\boldsymbol{B}_{\mathrm{r}}^{(l+1)}\left(\mathrm{r}_{l}, \zeta\right) \quad$ at allinterfaces

$\boldsymbol{H}_{\mathrm{r}}^{(l)}\left(\mathrm{r}_{l}, \zeta\right)=\boldsymbol{H}_{\mathrm{r}}^{(l+1)}\left(\mathrm{r}_{l}, \zeta\right)$ at interfaces without current sheets

$\boldsymbol{H}_{\mathrm{r}}^{(l)}\left(\mathrm{r}_{l}, \zeta\right)=\boldsymbol{H}_{\mathrm{r}}^{(l+1)}\left(\mathrm{r}_{l}, \zeta\right)+J_{l}(\zeta)$ at interfaces with current sheets

where $l=1, . ., 4$, with $r_{1}=r_{i, M}, r_{2}=r_{\mathrm{e}, M}, r_{3}=r_{S}$, $r_{4}=r_{S}+\Delta r_{b i}$, and $\mathbf{J}_{l}(\zeta)$ are the Fourier transform of the current sheets as defined in (2) and (3). Since the equivalent currents of the PMs are periodic, $\mathbf{J}_{1}(\zeta)$ and $\mathbf{J}_{2}(\zeta)$ are discrete functions of $\zeta$, with: $J_{1}\left(\frac{2 \pi}{\lambda} i\right)=J_{\mathrm{i}, 1} e^{j \varphi_{1,1}}$ and $J_{2}\left(\frac{2 \pi}{\lambda} i\right)=J_{i, 2} e^{j \varphi_{1,2}}$ as is (2). Considering $J_{3}(\zeta)$, we observe that it is a continuous function since $\boldsymbol{J}_{\text {stat }}(r, z, t)$ is not periodic along the axial direction. It can be discretized if the sampling interval is short enough to avoid spatial aliasing.
As known, to a sampling interval $\Delta \zeta$ corresponds a space replication of the waveform in (3), with a period of $2 \pi / \Delta \zeta$. Since $L$ is the length of the stator, we have to choose $\Delta \zeta<2 \pi / L$, and $J_{3}(i \Delta \zeta)=\frac{2}{k-i \Delta \zeta} \sin \left((k-i \Delta \zeta) \frac{L}{2}\right)$, with $k=2 \pi p / L$.

The algebraic system (13) is solved in correspondence of the values of the variable $\zeta$ which provide appreciably nonzero values of the corresponding spatial harmonics. The inverse Fourier transform is then performed to obtain the distribution of all the electromagnetic quantities.

The described semi analytical model is solved twice. The first time considering only the effects produced by the equivalent magnetization currents only, and the produced results are used to evaluate the no-load voltage. The second time considers the effects of the stator currents to evaluate the internal impedance of the machine.

\section{POST-PROCESSING}

Once the magnetic flux density distribution due to the travelling equivalent current distributions on the mover is known, we can evaluate the voltages at the terminals of the stator windings.

A line integration of the vector potential is performed on circular lines located on the current sheets and corresponding to the turns of the windings.

The circular lines and their orientations are determined by considering the position of the stator conductors inside the slots and the direction in which they are wound. The axial position of the turns of a coil ranges from the $z$ coordinates corresponding to the middle points of the adjacent teeth that delimit the slot where the coil is located. The evaluation of the coil-related voltage is then obtained by a double integral. The summation of the contributions of these integrals is performed according to the winding scheme:

$$
\begin{aligned}
\tilde{E}_{k, 0}^{M}= & j \omega \sum_{h=1}^{N_{\text {slot }}^{(k)}} \alpha_{h}^{(k)} N_{c o n d, h}^{(k)} \frac{1}{z_{2, h}^{(k)}-z_{1, h}^{(k)}} \int_{0}^{2 \pi} \int_{z_{1, h}^{(k)}}^{z_{2, h}^{(k)}} \tilde{A}_{\varphi}\left(r_{2}, z\right) r_{2} d \varphi d z= \\
& =j \omega 2 \pi r_{2} \sum_{h=1}^{N_{s l o t}^{(k)}} \alpha_{h}^{(k)} N_{c o n d, h}^{(k)} \frac{1}{z_{2, h}^{(k)}-z_{1, h}^{(k)}} \int_{z_{1, h}^{(k)}}^{z_{2, h}^{(k)}} \tilde{A}_{\varphi}\left(r_{2}, z\right) d z
\end{aligned}
$$

where the superscript $M$ in $\tilde{E}_{k, 0}^{M}$ indicates the action of the equivalent magnetization currents. In (14) $N_{\text {slot }}^{(k)}$ is the number of slots occupied by coils of the k-th phase winding and $N_{c o n d, h}^{(k)}$ is the number of conductors in the h-th slot of the k-th phase. The coefficient $\alpha_{h}^{(k)}$ assumes the values \pm 1 depending on the coil direction while $z_{1, h}^{(k)}$ and $z_{2, h}^{(k)}$ individuate the position of the h-th slot of the k-th phase.

Once the no-load voltage is obtained, to evaluate the internal impedance of the machine we solve the model 


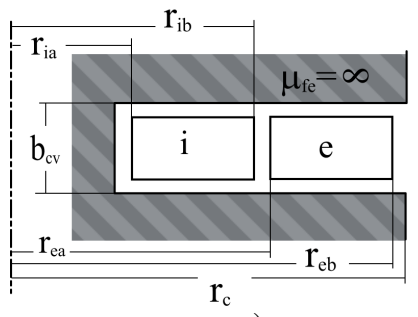

a)

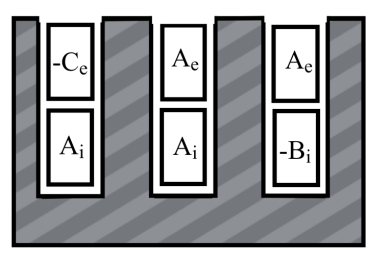

b)
Figure 6. Coil distribution in a slot and winding scheme (courtesy of [25]).

considering a current sheet on the stator corresponding to a three-phase balanced system of unit currents.

Equation (14) is evaluated again under this feeding condition, and it represents only a portion of the voltage at the terminal windings. Let us denote this contribution by $\tilde{E}_{k}^{(1)}$, where the superscript (1) refers to the action of the unit currents.

Considering the real arrangement of conductors in the slots, the effects of the voltage drops due to resistance of the conductors and to the leakage fluxes in the slots have to be considered.

A leakage impedance is introduced, whose resistive part is evaluated using the $\mathrm{d}$. c. values of the wires which constitute the windings, whereas the reactance is evaluated using the classical approach as in electrical machines textbooks [27].

The approach reported in [25] is here summarized. Let us consider Fig. 6a and assume that the magnetic field strength be non zero inside the slot only, and directed in the axial direction. Magnetic energy is written as:

$$
\begin{aligned}
W_{i / e}= & \mu_{0} \frac{N_{i / e}^{2} I_{i / e}^{2}}{b_{c v}}\left\{\frac { \pi } { ( r _ { i / e , b } - r _ { i / e , a } ) ^ { 2 } } \left[\frac{1}{4}\left(r_{i / e, b}^{4}-r_{i / e, a}^{4}\right)-\frac{2}{3} r_{i / e, a} .\right.\right. \\
& \left.\left.\left(r_{i / e, b}^{3}-r_{i / e, a}^{3}\right)+\frac{1}{2} r_{i / e, a}^{2}\left(r_{i / e, b}^{2}-r_{i / e, a}^{2}\right)\right]+\frac{\pi}{2}\left(r_{c}^{2}-r_{i / e, b}^{2}\right)\right\} \\
W_{i, e}= & \mu_{0} \frac{N_{i} I_{i} N_{e} I_{e}}{b_{c v}}\left\{\frac { 2 \pi } { r _ { e , b } - r _ { e , a } } \left[\frac{1}{3}\left(r_{e, b}^{3}-r_{e, a}^{3}\right)-\frac{1}{2} r_{e, a} .\right.\right. \\
& \left.\left.\left(r_{e, b}^{2}-r_{e, a}^{2}\right)\right]+\pi\left(r_{c}^{2}-r_{e, b}^{2}\right)\right\}
\end{aligned}
$$

and the self and mutual inductances are evaluated as:

$$
L_{i / e}=\frac{2 W_{i / e}}{I_{i / e}^{2}} \quad \text { and } \quad M_{i, e}=\frac{W_{i, e}}{I_{i} I_{e}} .
$$

Because of the winding constitution (ref. Fig. 2 and Fig. 6b) is:

$$
L_{\text {sect }}=2\left(L_{i}+L_{e}+M_{i, e}\right)
$$

When the machine is connected to a three-phase balanced load, a three-phase balanced system of currents flows on the windings. An equivalent self inductance coefficient taking into account the coupling with the other phases and evaluated as:

$$
L_{t o t}=N_{s e c t}\left(L_{s e c t}-M_{i, e} e^{-j 2 \frac{\pi}{3}}-M_{i, e} e^{-j 4 \frac{\pi}{3}}\right),
$$

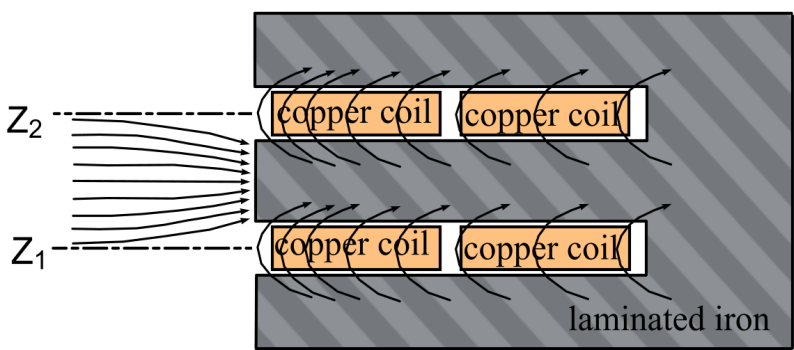

Figure 7. Composition of the flux density in a tooth (courtesy of [25]).

can be series connected to the above mentioned resistance. where $N_{\text {sect }}$ represents the number of series connected sections

(as in Fig. 3b) of a given phase that constitute the entire winding. For the shown device, $N_{\text {sect }}=4$.

Combining the leakage impedance and the $\tilde{E}_{k}^{(1)}$ the internal impedance of the machine is obtained.

Once a load connected to the generator is assigned, and the no load voltage and the internal impedance of the machine have been determined, the three-phase balanced system of currents on the windings can be evaluated.

The model described in Sect. II is used again with the system of current sheet equivalent to the currents just evaluated. Let $\tilde{B}_{r, p}\left(r_{k}, z\right)$ the flux density distribution obtained without considering the equivalent magnetization currents of the PMs and evaluated in correspondence of the position of the equivalent magnetization currents of the PMs $\left(r_{1}=r_{i, M}\right.$ and $\left.r_{2}=r_{\mathrm{e}, M}\right)$.

The model described in Sect. II can be used to determine the flux density distribution $\tilde{B}_{r, p}\left(r_{k}, z\right)$ produced by the current sheet which is equivalent to the three phases balanced currents on the stator, while the equivalent magnetization currents of the PMs are set to zero.

The average force on the mover in the axial direction can be evaluated by summing terms:

$F_{z, k}=\frac{1}{2} \int_{0}^{2 \pi} \int_{-L / 2}^{L / 2} \operatorname{Re}\left\{\tilde{J}_{\varphi, p}\left(r_{k}, z\right) \tilde{B}_{r, p}^{*}\left(r_{k}, z\right)\right\} d z d \varphi$

where $k$ spans the current sheets $\tilde{J}_{\varphi, p}\left(r_{k}, z\right)$ as shown in Fig. 5 (those due to the equivalent magnetization currents of the PM) and $p$ spans the harmonics as in (4). Using (18) implies discarding iron losses on the stator.

We also tried to extend the results by the semi-analytical model in order to obtain an estimate of local quantities in the stator. Because of the presence of the slots and teeth, comparison with the results by Finite Element analysis is not straightforward. Some post processing on the analytical results is required [25].

The flux density in a tooth corresponding to a given current on the stator windings can be considered as the sum of two terms. 
One term $\left(\boldsymbol{\Phi}_{a}\left(R_{S}\right)\right)$ "enters" the tooth from the surface facing the air gap; the other $\boldsymbol{\Phi}_{b}\left(R_{S}\right)$ "enters" the tooth from the adjacent slots. Fig 7 schematically shows how the flux lines compose in a stator tooth.

To evaluate the first contribution, the magnetic flux is computed through a cylindrical surface attached to the stator in correspondence of the tooth (the cylinder extends between the midpoints of the adjacent slots).

$$
\Phi_{a}\left(R_{S}\right)=\int_{0}^{2 \pi} \int_{z_{1}}^{z_{2}} \boldsymbol{B}_{r}\left(R_{S}, z\right) R_{S} d \vartheta d z
$$

where $\boldsymbol{B}_{r}\left(R_{S}, z\right)$ is the radial component of the flux density on the inner radius of the stator due to the equivalent magnetization currents and to the current sheet which is equivalent to the stator windings with the given current. The average flux density entering the tooth is obtained by dividing the flux as obtained by (19) by the surface of the tooth.

The second contribution, due to the leakage fluxes in the slots, is given by:

$$
\Phi_{b}(r)= \begin{cases}\varphi_{1}(r)=\frac{N_{e} I_{e}+N_{i} I_{i}}{b_{c}} \pi\left(r^{2}-r_{0}^{2}\right) & r_{0}<r<r_{1} \\ \varphi_{2}(r)=\varphi_{1}\left(r_{1}\right)+\frac{N_{e} I_{e}}{b_{c}} \pi\left(r^{2}-r_{1}^{2}\right)+ & r_{1}<r<r_{2} \\ +\frac{N_{i} I_{i}}{b_{c}\left(r_{2}-r_{1}\right)} 2 \pi\left[\frac{r_{2}}{2}\left(r^{2}-r_{1}^{2}\right)-\frac{\left(r^{3}-r_{1}^{3}\right)}{3}\right] & \\ \varphi_{3}(r)=\varphi_{2}\left(r_{2}\right)+\frac{N_{e} I_{e}}{b_{c}} \pi\left(r^{2}-r_{2}^{2}\right) & r_{2}<r<r_{3} \\ \varphi_{4}(r)=\varphi_{3}\left(r_{3}\right)+\frac{N_{e} I_{e}}{b_{c}\left(r_{4}-r_{3}\right)} 2 \pi . & \\ {\left[\frac{r_{4}}{2}\left(r^{2}-r_{3}^{2}\right)-\frac{\left(r^{3}-r_{3}^{3}\right)}{3}\right]} & \end{cases}
$$

\section{THE PROTOTYPE}

This section briefly describes a prototype available for some measurements to validate the proposed model. The geometry if the prototype is roughly the same as the one described in sect. II and the geometrical parameters are reported in table I.

As shown by Fig. 8, the most significant difference lies in the stator geometry. The prototype is not axisymmetric; as seen in the figure, it is made up of 8 modules that touch in the inner part (the one facing the translator) and diverge in the back iron. The reluctances of the paths in the iron stator are increased in the prototype with respect to those in the modelled machine; because of the relatively large airgap, the effects on the electrical quantities at the terminal of the machine are negligible.

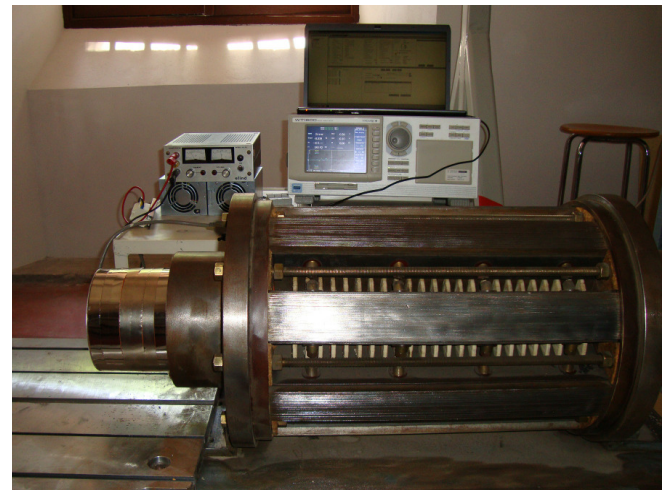

Figure 8. A picture of the prototype of the machine used to validate the model.

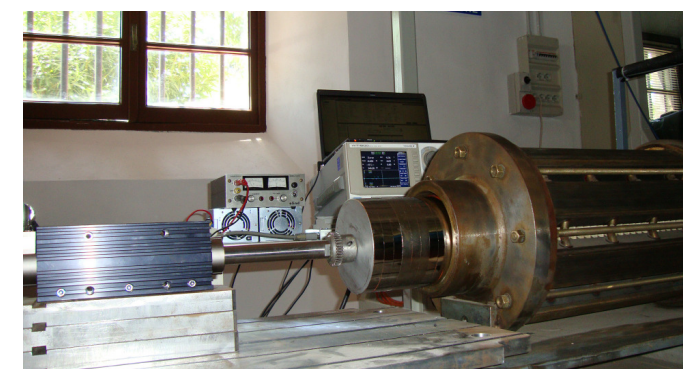

Figure 9. The prototype coupled with a linear motor.

The translator is $60 \mathrm{~cm}$ long, with a stroke of $25 \mathrm{~cm}$. The movement of the translator was obtained by a step linear motor capable of a nominal thrust force of $585 \mathrm{~N}$, with a maximum speed of $1.7 \mathrm{~m} / \mathrm{s}$ and a stroke of $21 \mathrm{~cm}$. Fig. 9 shows the test bench used to evaluate the no-load voltage, and the currents on a resistive load of $8.5 \Omega$.

\section{RESULTS}

This section shows the results produced by the proposed model to the analysis of the PMs linear tubular generator whose parameters are reported in Sect. II. Some experimental data are taken on the prototype described in the above section. A comparison with the numerical results obtained by a FEM analysis is reported too [28]. Where possible, comparisons between analytical, numerical and experimental results are reported.

The flux density distribution produced by the translator alone, i.e. without the presence of the stator, has been investigated firstly. Fig. 10 shows the waveform of the radial component of the magnetic flux distribution in two regions: inside the translator (at the mean radius of the annular region occupied by the PMs), and outside the translator (1.5 $\mathrm{mm}$ away in the radial direction).

Comparison between the analytical and numerical results has been performed in the first region, showing an excellent agreement. The origin of the $\mathrm{z}$-axis is taken in correspondence of the middle point of the fourth PM as in Fig. 3a. 


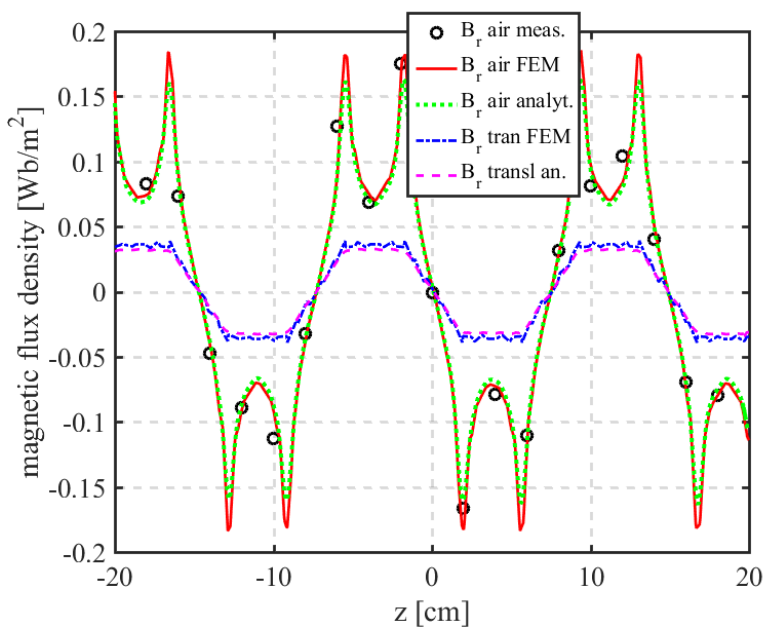

Figure 10. Radial components of the magnetic flux density produced by the PMs without the presence of the stator.

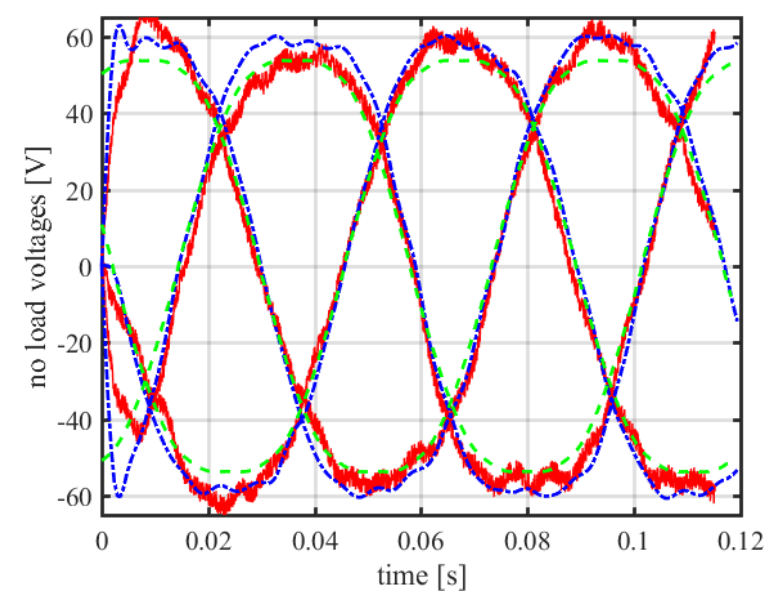

Figure 11. No load 3-phase voltage waveforms at the terminals of the machine: solid (red): measured, dashed (green): analytical, dashdot (blue): numerical.

In the second region, we compared the results obtained by the proposed model, the FEM analysis, and experimental measurements. In this case too, the agreement is fully satisfactory. Measurements have been performed by using a portable Gauss-meter F. W. Bell/4048 equipped with an accurate Hall sensor.

Fig. 11 shows the comparison between the numerical, analytical and experimental values of the no load voltages at the terminals of the machine. The measured quantities have been obtained by the experimental setup described in Sect. IV. The step linear motor was programmed for the maximum stroke $(21 \mathrm{~cm})$ at the maximum speed $(1.7 \mathrm{~m} / \mathrm{s})$. The measured voltages, recorded on a digital oscilloscope, are shown by the red lines. The blue lines refer to the numerical analysis, while the green ones are obtained by the analytical model, in particular by using (14). Both experimental and numerical results are affected by a ripple due to the presence of slots and teeth on the stator.

Fig. 12 reports the radial component of the magnetic flux density in the middle of the airgap of the machine at the noload condition, and when a three phase balanced load constituted by three resistors of $8.5 \Omega$ each, is star connected to the machine terminals. The results produced by the analytical model and by the finite elements analysis are compared at both the operating conditions. As described in sects. II and III the magnetic flux density at load condition is obtained by combining the no-load results with the effects of the currents which flow in the stator windings. The waveforms in Fig. 12 are obtained at $t=33 \mathrm{~ms}$, i.e. when the translator has moved of $5.6 \mathrm{~cm}$ with respect to the initial position which corresponds to the configuration in Fig. 10.

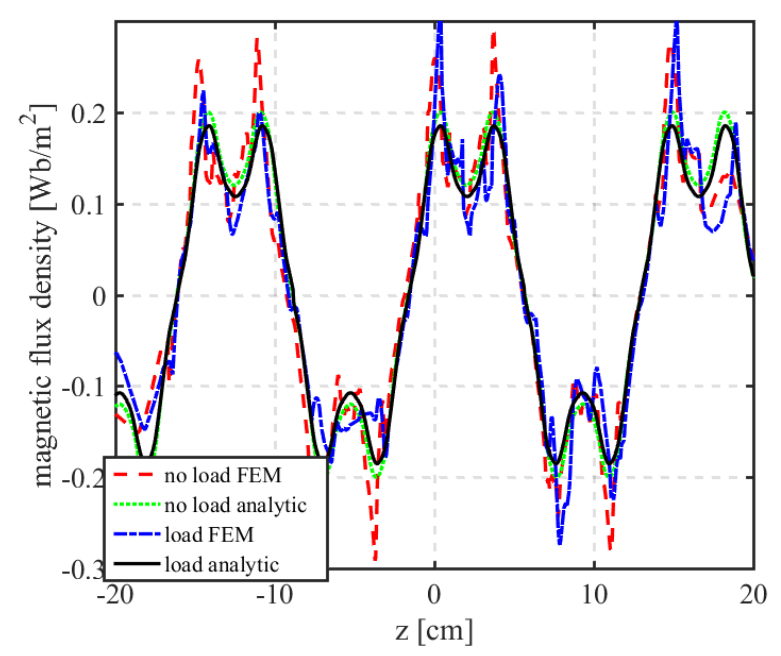

Figure 12. Comparison between the radial components on the magnetic flux density in the airgap under load and no load conditions evaluated by the analytical model and FEM.

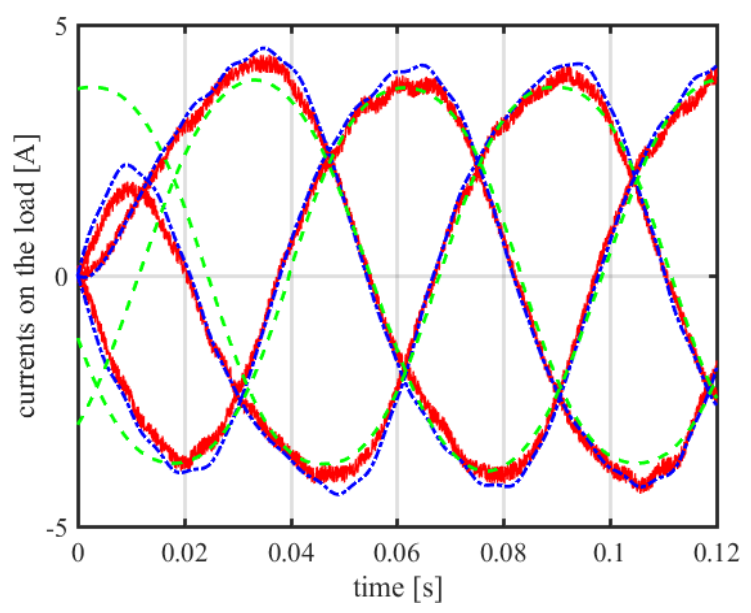

Figure 13. 3-phase current waveforms on the $8.5 \Omega$ resistive load; solid (red): measured, dashed (green): analytical, dashdot (blue): numerical. 
Because of the presence of the currents in the stator windings the magnetic flux density is slightly reduced with respect to the no load condition. The results by the numerical analysis are affected by a ripple which is produced by the slotted stator. As expected, the waveform produced by the analytical model are smooth.

Fig. 13 shows the comparison between the currents in the load evaluated by the numerical (blue lines) and the analytical model (green lines) and the experimental results (red lines) taken on the prototype. A ripple, due to the slotted structure of the stator, is observed in the numerical and experimental results.

As far the evaluation of the internal impedance per phase of the machine we considered the windings constituted by a copper wire with a cross section of $2.0 \mathrm{~mm}^{2}$; the total DC resistance of one winding is about $3.1 \Omega$ while according to (15) - (17) we obtained $L_{\text {tot }} \simeq 98 \mathrm{mH}$.

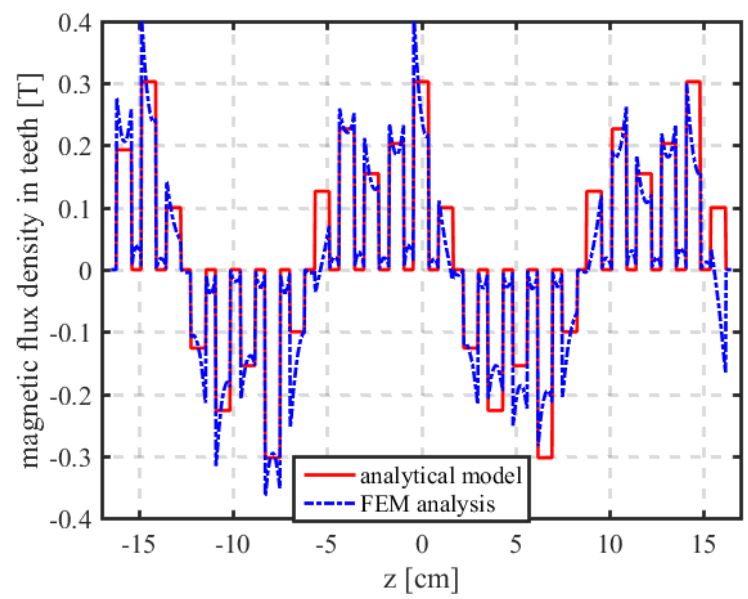

Figure 14. Comparison between the radial components of the magnetic flux just inside the stator teeth.

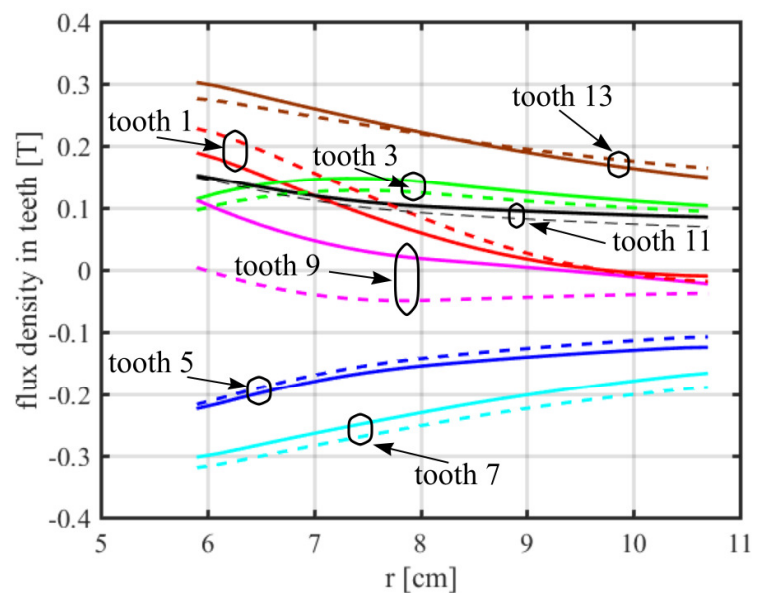

Figure 15. Waveforms of the mean value in the teeth of the radial component of the magnetic flux density. The solid lines refer to the semi-analytical model, the dashed ones to the finite elements analysis.
By using (19) the average flux density just inside the teeth of the stator $(r=56.5 \mathrm{~mm})$ has been evaluated. Fig. 14 shows the comparison between the results obtained by the proposed formulation with those by a FEM analysis. The analytical formulation is able to evaluate the average of the radial component only, while the FEM analysis allows evaluating the effective distribution in the cross section of the teeth. There is a good agreement between the results by the two methods, except in the rightmost teeth where the edge effects are more pronounced.

Fig. 15 reports the waveforms of the average values of the radial component of the magnetic flux density in some stator teeth as a function of the radial position. The generator operates under the above described load conditions. The tooth number 1 , as referred in the legend of Fig. 15, is the leftmost one in Fig. 14.

Finally, by (18) we evaluated the mean value of the force on the mover at the speed of $1.7 \mathrm{~m} / \mathrm{s}$ under the described load condition. The value obtained by the semi-analytical model was $162 \mathrm{~N}$ while the result of the Finite Element analysis was $173 \mathrm{~N}$.

\section{CONCLUSION}

Some preliminary results of the application of a semi analytical model to the analysis of a PMs linear tubular generator have been presented. The proposed formulation is able to perform the analysis of the machine under some simplifying hypotheses: a) substitution of the real stator with a smooth one by using the Carter factor, b) magnetization of the PMs is not affected by the armature reaction, c) indefinite extension of the machine along the axial direction. The results by the proposed model were compared with those obtained by finite element analysis and with experimental data taken on a prototype. The analytical model was able to produce accurate enough results in a short time. This makes the presented model a candidate tool for the automatic design and optimization of these class of generators.

\section{REFERENCES}

[1] A. Muetze and J. Vining, "Ocean wave energy conversion-A survey," in Conf. Rec. 41st IEEE IAS, Oct. 2006, vol. 3, pp. 1410-1417.

[2] A. Clément, P. McCullen, A. Falcao, A. Fiorentino, F. Gardner, K. Hammarlund, G. Lemonis, T. Lewis, K. Nielsen, S. Petroncini, M.-T. Pontes, P. Schild, B.-O. Sjostrom, H. C. Sorensen, and T. Thorpe, "Wave energy in Europe: Current status and perspectives," Renew. Sustain. Energy Rev., vol. 6, no. 5, pp. 405-431, Oct. 2002.

[3] V. Cecconi, V. Di Dio, A. O. Di Tommaso, S. Di Tommaso, D. La Cascia, R. Miceli, "Active power maximizing for wind electrical energy generating systems moved by a modular multiple blade fixed pitch wind turbine", SPEEDAM 2008 - International Symposium on Power Electronics, Electrical Drives, Automation and Motion, 2008, pp. 14601465 .

[4] G. Cipriani, M. Corpora, V. Di Dio, R. Miceli, C. Spataro, and M. Trapanese, "Technical and economical comparison between NdFeB and hard ferrites linear electrical generators from sea waves," in 2015 International Conference on Renewable Energy Research and Applications (ICRERA), 2015, pp. 1518-1522.

[5] Cipriani, G., Di Dio, V., La Manna, D., Massaro, F., Miceli, R., Zizzo, G., "Economic analysis on dynamic photovoltaic systems in new Italian "feed in tariffs" context", 4th International Conference on Clean Electrical Power: Renewable Energy Resources Impact, ICCEP 2013, pp. 584-590. 
[6] Battezzato A, G. Bracco, E. Giorgielli. G. Mattiazzo, Performance Assessment of a 2 DOF Gyroscopic Wave Energy Converter, Journal of Theoretical And Applied Mechanics, vol. 53, pp. 195-207, 2015.

[7] E. A. Amon, T. K. A. Brekken, A. A. Schacher. "Maximum power point tracking for ocean wave energy conversion." IEEE Transactions on Industry applications vol. 48, no. 3, pp.: 1079-1086, 2012.

[8] L. Cappelli, F. Marignetti, G. Mattiazzo, E. Giorcelli, G. Bracco, S. Carbone, C. Attaianese, "Linear tubular permanent-magnet generators for the inertial sea wave energy converter," IEEE Transactions on Industry Applications, vol. 50, no. 3, pp.: 1817-1828, 2014

[9] A. Souissi, M. W. Zouaghi, I. Abdennadher, A. Masmoudi, "MEC-based modeling and sizing of a tubular linear PM synchronous machine," IEEE Transactions on Industry Applications, vol. 51, no. 3, pp.: 2181-2194, 2015.

[10] http://www.seabased.com.

[11] J. Prudell, M. Stoddard, E. Amon, T. K. A. Brekken and A. von Jouanne, "A permanent-magnet tubular linear generator for ocean wave energy conversion," IEEE Trans. Ind. Appl., vol. 46, no. 6, pp. 23922400, Nov./Dec. 2010.

[12] V. D. Colli, P. Cancelliere, F. Marignetti, R. D. Stefano, and M. Scarano, "A tubular-generator drive for wave energy conversion," IEEE Trans. Industrial Electronics, vol. 53, no. 4, pp. 1152-1159. Apr 2006

[13] M. Leijon, H. Bernhoff, O. Ågren, J. Isberg, J. Sundberg, M. Berg, K. E. Karlsson, and A. Wolfbrandt, "Multiphysics Simulation of Wave Energy to Electric Energy Conversion by Permanent Magnet Linear Generator," IEEE Trans on Energy Conv. vol. 20, no. 1, pp. 219-224, Jan. 2005.

[14] V. Di Dio, and M. Montana, "State of the art of tubular linear induction motor," Proceeding of the Mediterranean Electrotechnical Conference, MELECON, Bari, 13-16 May 1996, pp. 285, 288.

[15] M. Trapanese, G. Cipriani, D. Curto, V. Di Dio, and V. Franzitta, "Optimization of cogging force in a linear permanent magnet generator for the conversion of sea waves energy," in Electric Machines Drives Conference (IEMDC), 2015 IEEE International, 2015, pp. 769-773

[16] G. Cipriani, V. Di Dio, V. Franzitta, A. Russo, M. Trapanese, and A. Viola, "A ferrite tubular linear permanent magnet generator (FTLPMG) analysis and design," in Oceans - St. John's, 2014, 2014, pp. 1-6.

[17] V. Boscaino, G. Cipriani, V. Di Dio, M. Corpora, D. Curto, V. Franzitta, and M. Trapanese, "The effect of manufacturing tolerances on a tubular linear ferrite motor," in Industrial Electronics Society, IECON 2015 41st Annual Conference of the IEEE, 2015, pp. 5174-5177.

[18] E. Cardelli, Advances in Magnetic Hysteresis Modeling, Handbook of Magnetic Materials, 24, pp. 323-409, 2015.

[19] E. Cardelli, A. Faba, A. Laudani, F. Riganti Fulginei, A. Salvini, "A neural approach for the numerical modeling of two-dimensional magnetic hysteresis", Journal of Applied Physics, vol. 117, no. 17 (2015), art. no. 17D129.

[20] S. Quondam Antonio, A. Faba, G. Carlotti, E. Cardelli, "Vector hysteresis model identification for iron-silicon thin films from micromagnetic simulations", Physica B: Condensed Matter, vol. 486, pp. 97-100, 2016.

[21] A. Musolino, R. Rizzo, M. Raugi, "A semi-analytical model for the analysis of a Permanent Magnet tubular linear generator," in Proc, 2015 IEEE International Conference on Renewable Energy Research and Applications (ICRERA), pp. 1513-1517.

[22] S. Barmada, A. Musolino, R. Rizzo, A. Tellini, "Fields analysis in axisymmetric actuators", IEEE Trans. Mag., vol. 36 n. 4, pp. 19061909, July 2000

[23] C. Liu, H. Yu, M. Hu, Q. Liu, S. Zhou, and L. Huang, "Research on a permanent magnet tubular linear generator for direct drive wave energy conversion." IET Renewable power generation vol. 8 no.3, pp.: 281-288, March 2013.

[24] J. Wang, G. W. Jewell and D. Howe, "A general framework for the analysis and design of tubular linear permanent magnet machines," IEEE Trans. Magn., vol. 35, no. 3, pp. 1986-2000, May 1999.

[25] A. Musolino, R. Rizzo, and E. Tripodi, "The double sided tubular linear induction motor and its possible use in the electro-magnetic aircraft launch system," IEEE Trans Plasma Sci., vol. 41, no. 5, pp. 1193-1200, May 2013

[26] W. P. Calixto, B. Alvarenga, A. P. Coimbra, A. J. Alves, L. M. Neto, M. Wu, W. G. da Silva and E. Delbone, "Carter's factor calculation using domain transformations and the finite element method," Int J. Num. Model. Electron. Network Dev. and Fields, vol. 25, n. 3, pp. 236-247, May 2012.

[27] M. Kostenko, and L. Piotrovski, Electrical Machines, vol. 1 and 2. : Moscow: Mir, 1968.

[28] MagNet V7, Infolytica Corporation, http://www.infolytica.com 